\title{
"Les enjeux d'une gestion territorialisée des risques technologiques. Spécificités françaises et mise en perspective internationale »
}

\section{Compte rendu de séminaire (Paris, novembre 2007 - janvier 2009)}

\author{
Jean-Pierre Galland, Emmanuel Martinais \\ 1 Sociologue, LATTS, Université Paris Est / École nationale des ponts et chaussées, 77455 Marne-la-Vallée cedex 2, France \\ 2 Géographe, RIVES, UMR 5600, CNRS / Université de Lyon / École nationale des travaux publics de l'État, \\ 69518 Vaulx-en-Velin cedex, France
}

Ce séminaire ${ }^{1}$ a eu comme origine une « commande», au printemps 2006, du Plan Urbanisme, Construction et Architecture (PUCA) du ministère de l'Équipement. À cette époque, la direction du PUCA, qui dépend de la direction générale de l'Urbanisme, de l'Habitat et de la Construction (DGUHC), prend acte du fait que la loi «Bachelot » du 30 juillet 2003 appelle une mobilisation importante et nouvelle des services déconcentrés de l'Équipement - les directions départementales et régionales (DDE et DRE) - sur un sujet qu'ils ne faisaient qu'effleurer jusque-là : le risque industriel. Pendant près de deux cents ans, en effet, la question des risques technologiques a surtout fait intervenir, du côté de l'État français, les inspecteurs des installations classées, réunis au sein des directions régionales de l'Industrie, de la Recherche et de l'Environnement (DRIRE). Mais la catastrophe d'AZF, à Toulouse en 2001, a révélé, une fois encore, les dangers d'une intrication mal maîtrisée entre la ville et ses industries. C'est pourquoi la loi du 30 juillet 2003, qui entend tirer les enseignements de cet événement, cherche à davantage « territorialiser » la prévention du risque industriel et à l'inscrire dans l'urbanisme, par le biais notamment du Plan de prévention des risques technologiques (PPRT).

\footnotetext{
Auteur correspondant : J.-P. Galland, galland@enpc.fr

1 Organisé par le PUCA, le laboratoire Techniques, Territoires et Sociétés (LATTS) et le laboratoire de recherches interdisciplinaires Ville, Espace, Société (RIVES) de l’ENTPE, il a lieu au MEEDDAT (La Défense).
}

Au-delà, cette loi innove sur au moins quatre points :

- à l'instar des risques naturels, le nouveau cadre réglementaire impose tout d'abord une conception du risque industriel en termes d'aléas (technologiques) et de vulnérabilités (territoriales), ce qui nécessite d'identifier précisément les «enjeux » locaux et leur sensibilité aux aléas préalablement à la mise en règlement $\mathrm{du}$ PPRT;

- les dispositions susceptibles d'être retenues pour réduire la vulnérabilité de ces « enjeux » ne portent plus uniquement sur l'urbanisation future des territoires menacés, mais également sur l'urbanisation existante;

- ces dispositions (expropriation et délaissement) doivent être financées et mises en œuvre dans le cadre de partenariats locaux originaux, impliquant au cas par cas l'État, l'industriel et la collectivité locale concernés, l'intérêt de telle ou telle action sur l'habitat «vulnérable » devant au préalable être mis en regard d'une solution de réduction du risque «à la source » dans l'entreprise à l'origine du PPRT ;

- enfin, la loi instaure la participation d'un ensemble plus ou moins large d'acteurs locaux au travail de production de la règle de droit, dans le cadre d'une procédure d'élaboration conçue comme démocratique et transparente, notamment à travers la mise en place de comités locaux d'information et de concertation (CLIC).

En raison de ces importantes évolutions réglementaires, les $\mathrm{D}(\mathrm{R}) \mathrm{DE}$ se sont vues confier, aux côtés des DRIRE, la coresponsabilité de la mise en œuvre de cette 
nouvelle politique de maîtrise des risques industriels, avec un objectif ambitieux de réalisation de PPRT en nombre.

$C^{\prime}$ est dans ce contexte qu'a été rédigé, à la demande des commanditaires ministériels qui s'interrogeaient sur les effets de cette réforme, un texte de problématique et qu'a été mis sur pied un séminaire d'échanges entre praticiens et chercheurs. Au-delà d'une attention particulière portée à la «montée en puissance » des $\mathrm{D}(\mathrm{R}) \mathrm{DE}$ sur la question des risques industriels, ce séminaire vise à examiner dans toutes leurs dimensions et dans toutes leurs interactions les multiples évolutions (méthodologiques, techniques, économiques, sociales) suscitées par cette réforme de la politique française de prévention des risques industriels, tout en essayant de prendre du champ par rapport au vieux problème des rapports entre l'industrie et le territoire, notamment par le moyen de comparaisons internationales.

Les sept séances du séminaire ont eu lieu entre novembre 2007 et janvier 2009. La première a permis de revenir sur les «fondamentaux » de la prévention des risques industriels en France, sur le rôle historique de l'Inspection des installations classées, ainsi que sur la genèse de la loi Bachelot, notamment à travers le témoignage de l'un de ses rédacteurs. Les deux séances suivantes ont été consacrées aux outils de l'analyse des risques industriels, que ce soit du côté des «sources » (discussion autour des «études de dangers » et de la "caractérisation des aléas technologiques ») ou du côté des « cibles » : comment caractériser la vulnérabilité d'un territoire. Les $4^{\mathrm{e}}$ et $5^{\mathrm{e}}$ séances ont davantage porté sur les questions de concertation et de démocratie locale, en premier lieu à propos des capacités des agents des services déconcentrés de l'État à organiser et prendre en compte cette concertation, puis à travers une interrogation sur les difficultés de certaines parties prenantes (riverains, associations de défense de l'environnement, salariés des industries concernées) à intervenir lors des discussions organisées dans le cadre des comités pourtant légalement instaurés. Les $6^{\mathrm{e}}$ et $7^{\mathrm{e}}$ séances ont respectivement porté sur les aspects économiques de la gestion du risque industriel et sur une ouverture à d'autres pratiques en Europe.

Ce séminaire a d'emblée été conçu et voulu comme un lieu de discussion et de confrontation entre praticiens et chercheurs. Chaque séance, qui dure une courte journée, donne lieu à un nombre limité d'interventions formelles, une large place étant réservée aux débats. Le public (une cinquantaine de personnes en moyenne, venues bien souvent des divers coins de l'Hexagone) est largement composé de représentants des services déconcentrés de l'État (DDE majoritairement, mais aussi DRIRE), de représentants de certaines collectivités locales particulièrement concernées par le risque industriel, mais aussi de chercheurs spécialisés dans ces questions. Les intervenants sont aussi divers que peuvent l'être les intérêts et les compétences en jeu. Du côté des praticiens, les inspecteurs des installations classées, les représentants des DDE ou d'autres services liés à l'ex-ministère de l'Équipement (Centre d'études techniques de l'Équipement - CETE) se sont largement exprimés. Des maires, des industriels, des associations de défense de l'environnement, des riverains et des salariés sont également intervenus. Du côté académique, la palette est également large, entre le point de vue des ingénieurs fiabilistes de l'Institut national de l'environnement industriel et des risques (INERIS), celui des géographes ou autres spécialistes des systèmes d'information géographique, celui des économistes férus d'analyse coût/bénéfice ou celui, enfin, de sociologues ou de politistes. Par ailleurs, l'actualité du sujet lui-même (la mise en œuvre de la loi Bachelot, et en particulier des PPRT) a conduit à confronter, souvent au sein d'une même séance, discours théoriques et témoignages ou études de cas.

Il est trop tôt pour tirer un bilan d'une action de recherche à peine achevée. D'ores et déjà, on peut cependant essayer de dégager une esquisse de conclusion, sous forme d'hypothèses à approfondir, à la fois à l'usage des commanditaires (lesquels demandent, à travers ce séminaire, de mettre en exergue les «bonnes pratiques » et de dessiner de nouvelles pistes de recherche) et, plus généralement, de la communauté scientifique.

Côté «bonnes pratiques ", ce séminaire témoigne finalement de la grande capacité des représentants des services (techniques) déconcentrés de l'État à recomposer leur action à la suite de changements réglementaires majeurs. Après quelques incompréhensions, voire quelques notables tensions, les DRIRE et les $\mathrm{D}(\mathrm{R}) \mathrm{DE}$ mènent désormais de concert bon nombre d'actions sur le terrain, tentent d'intégrer à leurs décisions les contraintes et les ressources de la démocratie locale. Bref, elles semblent prêtes à traduire au mieux l'ambitieuse philosophie de la loi sur le terrain. Les conditions de cette coopération ont évolué au fil des événements politiques et administratifs de l'année 2007, jusqu'au regroupement des ex-ministères de l'Équipement et de l'Écologie au sein de l'actuel ministère de l'Écologie, de l'Énergie, du Développement durable et de l'Aménagement du territoire, dont dépendent désormais à la fois les $\mathrm{D}(\mathrm{R}) \mathrm{DE}$ et les DRIRE. Ainsi, à la différence d'autres États européens qui gèrent ces questions de plus en plus «à distance », la France apparaît plutôt comme le lieu d'un retour de l'État, par l'intermédiaire de ses services déconcentrés, en tout cas sur les territoires concernés par le risque industriel. Mais ce n'est pas tant l'État « régalien » qui revient sur ces territoires, à l'occasion de la mise en œuvre des PPRT, qu'un État à la fois pédagogue, calculateur et, éventuellement, animateur du débat public. Reste à tester cette hypothèse en entrant plus dans le détail de la comparaison internationale en matière de prise en compte des risques industriels. Mais aussi en mettant en perspective, cette fois sur le territoire national, l'éventuelle évolution du rôle de l'État et de 
ses services déconcentrés vis-à-vis de la prise en compte d'autres « risques » ou d'autres problèmes se situant dans ce registre de la sécurité publique.

Le texte problématique du séminaire, les programmes particuliers de ses diverses séances, ainsi que les comptes rendus des toutes premières sont disponibles sur le site Internet du PUCA : http://rp.urbanisme.equipement.gouv. fr/puca/agenda/frame_agenda.htm, rubrique "Agenda », à la date du 16 janvier 2009 , date de la $7^{\mathrm{e}}$ et dernière séance du séminaire. 